\title{
GLOBAL WEAK SOLUTIONS TO THE RELATIVISTIC VLASOV-MAXWELL SYSTEM REVISITED *
}

\author{
GERHARD REIN ${ }^{\dagger}$
}

\begin{abstract}
In their seminal work [3], R. DiPerna and P.-L. Lions established the existence of global weak solutions to the Vlasov-Maxwell system. In the present notes we give a somewhat simplified proof of this result for the relativistic version of this system, the main purpose being to make this important result of kinetic theory more easily accessible to newcomers in the field. We show that the weak solutions preserve the total charge.
\end{abstract}

Key words. Relativistic Vlasov-Maxwell system; global weak solutions; collisionless plasma.

\section{Introduction}

When a plasma is sufficiently rarefied and/or sufficiently hot like in the solar wind or in a powered-up fusion reactor collisions among the plasma particles are sufficiently rare to be neglected. The only interaction among the particles then is through the electromagnetic fields which the particles create collectively. For the sake of simplicity we restrict ourselves to a plasma consisting of just one particle species, say, electrons, and we allow for the possibility that the particles move at relativistic speeds. The time evolution of the plasma is governed by the relativistic Vlasov-Maxwell system:

$$
\begin{gathered}
\partial_{t} f+\widehat{p} \cdot \partial_{x} f+(E+\widehat{p} \times B) \cdot \partial_{p} f=0, \\
\partial_{t} E-\operatorname{curl} B=-4 \pi j, \quad \partial_{t} B+\operatorname{curl} E=0, \\
\operatorname{div} E=4 \pi \rho, \quad \operatorname{div} B=0, \\
\rho(t, x)=\int f(t, x, p) d p, \quad j(t, x)=\int \widehat{p} f(t, x, p) d p .
\end{gathered}
$$

Here $f=f(t, x, p)$ denotes the density of the particles on phase space, $t \in \mathbb{R}, x, p \in \mathbb{R}^{3}$ stand for time, position, and momentum,

$$
\widehat{p}:=\frac{p}{\sqrt{1+|p|^{2}}}
$$

is the velocity of a particle with momentum $p, E=E(t, x)$ and $B=B(t, x)$ are the electromagnetic fields, $\rho=\rho(t, x)$ and $j=j(t, x)$ denote the spatial charge density and current, and units are chosen such that all physical constants such as the speed of light and the charge and rest mass of an individual particle are normalized to unity. The analysis can immediately be adapted to a plasma with several species of particles.

We are interested in the Cauchy problem for the above system, i.e., in the existence of solutions satisfying the initial conditions

$$
f_{\mid t=0}=\stackrel{\circ}{f}, E_{\mid t=0}=\stackrel{\circ}{E}, B_{\mid t=0}=\stackrel{\circ}{B}
$$

*Received: March 2, 2004; accepted (in revised version): April 22, 2004. Communicated by Francois Golse.

$\dagger$ University of Bayreuth, Department of Mathematics, D-95440 Bayreuth, Germany (gerhard.rein@uni-bayreuth.de). 
where the initial data satisfy the constraint part (1.3) of the Maxwell equations. Global existence and uniqueness of sufficiently smooth solutions to this initial value problem is an open problem. Local existence and uniqueness of classical solutions for smooth, compactly supported data was established in [12]. These solutions can be extended globally in time provided the momentum support can be controlled, which has been done for data which are small [13] or close to neutral [7] or close to spherically symmetric [22]. In lower dimensions global classical solutions exist for general data $[8,9,10,11]$. Different approaches to the result in [12] were recently given in $[1,16]$. There is as yet no indication that classical solutions for general data in three dimensions develop singularities. Nevertheless, it is natural to weaken the solution concept in order to obtain global solutions. This was done by R. DiPerna and P.L. Lions [3]. The authors restricted themselves to the non-relativistic Vlasov-Maxwell system where $\widehat{p}$ is replaced by $p$ and pointed out that their arguments apply to the relativistic case as well. Many results on the Vlasov-Maxwell system are reviewed in the monograph [5], in particular, global weak solutions are discussed following [3], but using also arguments introduced in [17]. The techniques in [3] are closely related to those used by the same authors in their seminal work on the Boltzmann equation [4], and later these techniques have been adapted to a variety of other problems in kinetic theory; we mention $[2,19]$ as being related to the present notes.

Given the fact that the global existence and uniqueness problem for the VlasovMaxwell system in three dimensions is still open and the fact that in recent years many young researchers have joined the field it should be useful to give a simplified proof of the result of DiPerna and Lions. We emphasize that all the essential techniques we are going to use are introduced in [3], but some non-trivial concepts and arguments from [3] are avoided. We concentrate on the relativistic version of the system, firstly, because being Lorentz invariant it seems better justified from a physics point of view, and secondly, because the non-relativistic case was considered in detail in $[3,5]$. In passing we note that there are global existence results for classical solutions of the Vlasov-Poisson system [21, 20, 23] but not yet for the so-called relativistic VlasovPoisson system where $p$ is replaced by $\widehat{p}$ in the Vlasov equation, cf. [6].

We now discuss how the paper and the proof proceed and how our version differs from the original one. In the next section we recall various a-priori bounds resulting from conservation of energy and conservation of phase space volume by the characteristic flow of the Vlasov equation. Then we introduce a regularized version of the system which has global in time, smooth solutions. It will be important that these regularized solutions exist on the whole time axis. In [3] the system was regularized by adding a sufficiently large power of the Laplacian to the evolution part of the Maxwell equations (1.2). This destroys the time reversibility of the system and technically impedes the straight forward application of the velocity averaging lemma discussed below. We propose to regularize the system by smoothing the current $j$ so that conceptually we remain closer to the unmodified system. This regularization is due to [15] and was used in the context of weak solutions in $[5,17,18]$, but its technical advantages were not fully realized. Given a sequence of solutions to regularized Vlasov-Maxwell systems along which the regularization vanishes in the limit we show that the a-priori bounds hold uniformly. Hence we can extract a weakly convergent subsequence whose limit is the candidate for the desired weak solution of the unmodified system. The main difficulty lies in passing to the limit in the nonlinear term in the Vlasov equation. This difficulty can be overcome since additional compactness of the approximating sequence is provided by the velocity averaging lemma. Its application 
is discussed in Section 5, and to make the present notes self-contained we give a proof of the relevant relativistic version in an appendix. Since the proof rests on Fourier transforming the Vlasov equation with respect to space and time it is advantageous that our approximating solutions are defined on the whole time axis. In the set-up of [3] a certain cut-off and extension maneuver was necessary. A second problem lies in passing to the limit in moments of $f$ like $\rho$ and $j$, the difficulty being that the relevant weights in $p$ are not test functions. As opposed to [3] we derive weak convergence of $\rho$ and $j$ directly from a-priori bounds for these quantities. In all this a minimal requirement is that the initial data have finite energy and finite total charge. The original proof assumed in addition that $\stackrel{\circ}{f}$ is square integrable. We assume that $f$ is bounded, and we completely avoid the non-trivial concept of renormalization which was necessary in [3] - in the opinion of the present author the main motivation for studying weak solutions is not to allow for the greatest possible generality in the initial data but the lack of global existence results for stronger solution concepts. Having obtained a weak solution we examine some of its properties in Section 6. Sufficient regularity is established to make sense of saying that the solution satisfies the initial conditions. Then we prove that for almost all times $t$ the total charge and more generally any $L^{q}$-norm of $f(t)$ equals its initial value, a result which was not obtained in [3] and for which we exploit the relativistic nature of the system. Since the Vlasov equation is a conservation law on phase space it is a desirable feature of any "reasonable" solution concept that solutions preserve the total charge. The energy at times $t \neq 0$ is bounded by its initial value but is not known to be conserved, and neither are weak solutions known to be unique.

To sum up, our proof is simplified compared to [3] in the sense that the only nontrivial tool employed is velocity averaging. The rest of the proof consists of straight forward exploitation of straight forward a-priori bounds.

\section{Preliminaries and a-priori bounds}

We introduce the main conservation laws for the relativistic Vlasov-Maxwell system; the derivations are formal, and in which sense they hold depends on the type of solution under consideration. Writing the Vlasov equation (1.1) in divergence form

$$
\partial_{t} f+\operatorname{div}_{x}[\widehat{p} f]+\operatorname{div}_{p}[(E(t, x)+\widehat{p} \times B(t, x)) f]=0
$$

and integrating with respect to $p$ yields local conservation of charge,

$$
\partial_{t} \rho+\operatorname{div}_{x} j=0 .
$$

On the level of the solutions of the characteristic system

$$
\dot{x}=\widehat{p}, \quad \dot{p}=E(t, x)+\widehat{p} \times B(t, x)
$$

of the Vlasov equation, conservation of charge is reflected in the induced flow on phase space being measure preserving and $f$ being constant along the flow. A lengthy computation shows local conservation of energy,

$$
\partial_{t} e+\operatorname{div}_{x} \sigma=0
$$

where the corresponding energy density and flux are defined by

$$
\begin{aligned}
e(t, x) & :=\int \sqrt{1+|p|^{2}} f(t, x, p) d p+\frac{1}{8 \pi}\left(|E(t, x)|^{2}+|B(t, x)|^{2}\right), \\
\sigma(t, x) & :=\int p f(t, x, p) d p+\frac{1}{4 \pi} E(t, x) \times B(t, x) .
\end{aligned}
$$


These local conservation laws imply corresponding global conservation laws:

Proposition 1. Consider a classical solution of the relativistic Vlasov-Maxwell system with compactly supported initial data $\stackrel{\circ}{f} \in C_{c}^{1}\left(\mathbb{R}^{6}\right), \stackrel{\circ}{f} \geq 0, \stackrel{\circ}{E}, \stackrel{\circ}{B} \in C_{c}^{2}\left(\mathbb{R}^{3}\right)$, satisfying the constraints (1.3). As long as the solution exists its energy

$$
\iint \sqrt{1+|p|^{2}} f(t, x, p) d p d x+\frac{1}{8 \pi} \int\left(|E(t, x)|^{2}+|B(t, x)|^{2}\right) d x
$$

as well as any $L^{q}$-norm $\|f(t)\|_{q}$ with $1 \leq q \leq \infty$ are constant in time.

We use the conserved quantities to derive bounds on $\rho$ and $j$ : For any $R>0$,

$$
\begin{aligned}
\rho(t, x) & =\int_{|p| \leq R} f(t, x, p) d p+\int_{|p|>R} f(t, x, p) d p \\
& \leq \frac{4 \pi}{3} R^{3}\|f(t)\|_{\infty}+R^{-1} \int \sqrt{1+|p|^{2}} f d p \leq C\left(\int \sqrt{1+|p|^{2}} f d p\right)^{3 / 4},
\end{aligned}
$$

where for the last step we choose

$$
R=\left(\int \sqrt{1+|p|^{2}} f d p\right)^{1 / 4}
$$

and the constant depends on $\|\dot{f}\|_{\infty}$. Taking both sides of the estimate to the power $4 / 3$, integrating in $x$, and using Proposition 1 we have the following a-priori bounds on $\rho$ and hence also $j$ which is dominated by $\rho$ :

Proposition 2. Along any solution as considered in Proposition 1,

$$
\|\rho(t)\|_{4 / 3},\|j(t)\|_{4 / 3} \leq C
$$

where the constant $C$ depends on the energy of the initial data and on $\|\stackrel{f}{f}\|_{\infty}$.

To conclude we observe that the constraints (1.3) propagate: if we have a solution of the system (1.1), (1.2), (1.4) satisfying the constraints (1.3) initially then the constraints hold as long as the solution exists, since

$$
\begin{gathered}
\partial_{t}\left(\operatorname{div}_{x} E-4 \pi \rho\right)=\operatorname{div}_{x} \partial_{t} E-4 \pi \partial_{t} \rho=\operatorname{div}_{x}\left(\partial_{t} E+4 \pi j\right)=\operatorname{div}_{x}\left(\operatorname{curl}_{x} B\right)=0 \\
\partial_{t} \operatorname{div}_{x} B=-\operatorname{div}_{x}\left(\operatorname{curl}_{x} E\right)=0 .
\end{gathered}
$$

\section{The regularized system}

For a mollifier

$$
d \in C_{c}^{\infty}\left(\mathbb{R}^{3}\right), d \geq 0, \int d=1, d \text { even }
$$

we consider the regularized relativistic Vlasov-Maxwell system (1.1), (1.2), (1.4) where in the Maxwell equations (1.2) we replace $j$ by $d * j$, the convolution referring to $x$; recall that the constraints (1.3) propagate once they hold initially. Along a (local) solution of the regularized system charge is still conserved, hence $\int|j(t)| \leq \int \rho(t) \leq$ $C$ and therefore all spatial derivatives of $d * j$ are bounded uniformly in $t$. This is sufficient to show that the regularized system has global-in-time solutions for initial data as specified in Proposition 1; details of the proof can be found in [15]. However, 
there is one difficulty with the regularized system: the time derivative of the energy is

$$
\int(j \cdot E-(d * j) \cdot E) d x
$$

which need not vanish. Hence, when constructing a sequence of approximating solutions by the regularization above we have to make sure we preserve the a-priori bounds, uniformly along the sequence. Let

$$
L_{\text {kin }}^{1}\left(\mathbb{R}^{6}\right):=\left\{g \in L^{1}\left(\mathbb{R}^{6}\right) \mid g \geq 0, \iint \sqrt{1+|p|^{2}} g(x, p) d p d x<\infty\right\}
$$

be endowed with the weighted $L^{1}$-norm $\|\cdot\|_{\text {kin }}$ with weight $\sqrt{1+|p|^{2}}$. We fix initial data

$$
\stackrel{\circ}{f} \in L_{\mathrm{kin}}^{1} \cap L^{\infty}\left(\mathbb{R}^{6}\right), \stackrel{\circ}{E}, \stackrel{\circ}{B} \in L^{2}\left(\mathbb{R}^{3}\right)
$$

satisfying the constraint (1.3) in the sense of distributions, and take sequences $\left(\stackrel{\circ}{n}_{n}\right) \subset$ $C_{c}^{\infty}\left(\mathbb{R}^{6}\right)$ and $\left(\stackrel{\circ}{E_{n}^{\sim}}\right),\left(\stackrel{\circ}{B_{n}^{\sim}}\right) \subset C_{c}^{\infty}\left(\mathbb{R}^{3}\right)$ such that

$$
\stackrel{\circ}{f}_{n} \rightarrow \stackrel{\circ}{f} \text { in } L_{\mathrm{kin}}^{1} \cap L^{\infty}\left(\mathbb{R}^{6}\right), \quad \stackrel{\circ}{E}_{n}^{\sim} \rightarrow \stackrel{\circ}{E}, \stackrel{\circ}{\stackrel{\sim}{*}_{n}^{\sim}} \rightarrow \stackrel{\circ}{B} \text { in } L^{2}\left(\mathbb{R}^{3}\right) .
$$

With $d$ as above we let $d_{n}(x):=n^{3} d(n x)$, define

$$
\stackrel{\circ}{E}_{n}:=d_{n} * \stackrel{\circ}{E}_{n}^{\sim} \rightarrow \stackrel{\circ}{E}, \stackrel{\circ}{B}_{n}:=d_{n} * \stackrel{\circ}{B}_{n}^{\sim} \rightarrow \stackrel{\circ}{B} \text { in } L^{2}\left(\mathbb{R}^{3}\right),
$$

and denote by $\left(f_{n}, E_{n}, B_{n}\right)$ the global solution of the regularized system with the initial data $\left(\stackrel{\circ}{f}_{n}, \stackrel{\circ}{E}_{n}, \stackrel{\circ}{B}_{n}\right)$, where $j$ now is replaced by $d_{n} * d_{n} * j_{n}$ - the two $d_{n}$ 's are intentional-with $j_{n}$ defined in terms of $f_{n}$ as in (1.4). This solution exists for all $t \in \mathbb{R}$ by the reasoning above. By uniqueness,

$$
\left(E_{n}, B_{n}\right)(t)=d_{n} *\left(E_{n}^{\sim}, B_{n}^{\sim}\right)(t)
$$

where $\left(E_{n}^{\sim}, B_{n}^{\sim}\right)$ solves the Maxwell equations $(1.2)$ with initial data $\stackrel{\circ}{E}_{n}^{\sim}, \stackrel{\circ}{B_{n}^{\sim}}$ and current $d_{n} * j_{n}$. The energy

$$
\iint \sqrt{1+|p|^{2}} f_{n}(t, x, p) d p d x+\frac{1}{8 \pi} \int\left(\left|E_{n}^{\sim}(t, x)\right|^{2}+\left|B_{n}^{\sim}(t, x)\right|^{2}\right) d x
$$

now is constant in time, its time derivative becoming

$$
\int\left(j_{n} \cdot E_{n}-\left(d_{n} * j_{n}\right) \cdot E_{n}^{\sim}\right) d x=0
$$

Since the modified energy defined above dominates the energy of the regularized solution we can use the arguments in the previous section to prove the following result, cf. Proposition 2:

Proposition 3. For initial data as specified in (3.2) there exists a constant $C>0$ such that for any $n \in \mathbb{N}$ the solution $\left(f_{n}, E_{n}, B_{n}\right)$ of the regularized initial value problem described above exists for all $t \in \mathbb{R}$ and satisfies the a-priori bounds

$$
\left\|f_{n}(t)\right\|_{\text {kin }},\left\|f_{n}(t)\right\|_{\infty},\left\|E_{n}(t)\right\|_{2},\left\|B_{n}(t)\right\|_{2},\left\|\rho_{n}(t)\right\|_{4 / 3},\left\|j_{n}(t)\right\|_{4 / 3} \leq C .
$$




\section{The weak limit}

Proposition 4. There exist functions

$$
f \in L^{\infty}\left(\mathbb{R} ; L_{\mathrm{kin}}^{1} \cap L^{\infty}\left(\mathbb{R}^{6}\right)\right), E, B \in L^{\infty}\left(\mathbb{R} ; L^{2}\left(\mathbb{R}^{3}\right)\right), \rho, j \in L^{\infty}\left(\mathbb{R} ; L^{4 / 3}\left(\mathbb{R}^{3}\right)\right)
$$

such that up to a subsequence

$$
f_{n} \rightarrow f \text { in } L^{2}\left(I \times \mathbb{R}^{6}\right), E_{n}, B_{n} \rightarrow E, B \text { in } L^{2}\left(I \times \mathbb{R}^{3}\right), \rho_{n}, j_{n} \rightarrow \rho, j \text { in } L^{4 / 3}\left(I \times \mathbb{R}^{3}\right)
$$

for any bounded interval $I \subset \mathbb{R}$,

$$
f \geq 0, \rho=\int f d p, j=\int \widehat{p} f d p \text { a. e., }
$$

and the Maxwell equations (1.2), (1.3) as well as local conservation of charge (2.2) hold in the sense of distributions. For almost all $t \in \mathbb{R}$ the energy of $(f(t), E(t), B(t))$ is bounded by its initial value.

Proof. The extraction of the weakly convergent subsequence is standard, and by a diagonal sequence argument this subsequence can be chosen independently of the interval $I$. Since $f_{n} \geq 0$ for $n \in \mathbb{N}$, the weak limit $f$ is non-negative almost everywhere. That the limits lie in the asserted function spaces is straight forward. For example let $A \subset \mathbb{R}$ be bounded and measurable with Lebesgue measure $\lambda(A)$, and let $R>0$. Then

$$
\begin{aligned}
& \int_{A} \int_{|x| \leq R}\left(\int_{|p| \leq R} \sqrt{1+|p|^{2}} f d p+\frac{1}{8 \pi}\left(|E|^{2}+|B|^{2}\right)\right) d x d t \\
& \quad=\lim _{n \rightarrow \infty} \int_{A} \int_{|x| \leq R}\left(\int_{|p| \leq R} \sqrt{1+|p|^{2}} f_{n} d p+\frac{1}{8 \pi}\left(\left|E_{n}\right|^{2}+\left|B_{n}\right|^{2}\right)\right) d x d t \\
& \leq \lambda(A)\left(\|\stackrel{\circ}{f}\|_{\text {kin }}+\frac{1}{8 \pi}\left(\|\stackrel{\circ}{E}\|_{2}^{2}+\|\stackrel{\circ}{B}\|_{2}^{2}\right)\right) .
\end{aligned}
$$

Since $R>0$ and $A \subset \mathbb{R}$ are arbitrary the assertion on the energy follows, which implies part of the assertion on the weak limits $f, E, B$.

For the moments $\rho$ and $j$ we can argue similarly, but we have to make sure that the weak limit of $\rho_{n}$ is the density $\rho$ induced by $f$ and analogously for $j$. To this end, consider a test function $\psi \in C_{c}^{\infty}\left(\mathbb{R} \times \mathbb{R}^{3}\right)$ and some $R>0$. Then

$$
\begin{aligned}
\iint & \left(\int \widehat{p} f d p-j\right) \psi d x d t \\
& =\iint\left(\int_{|p| \leq R} \widehat{p} f d p-j\right) \psi d x d t+\iiint_{|p|>R} \widehat{p} f d p \psi d x d t \\
& =\lim _{n \rightarrow \infty} \iint\left(\int_{|p| \leq R} \widehat{p} f_{n} d p-j_{n}\right) \psi d x d t+\iiint_{|p|>R} \widehat{p} f d p \psi d x d t \\
& =\lim _{n \rightarrow \infty} \iiint_{|p|>R} \widehat{p}\left(f-f_{n}\right) d p \psi d x d t .
\end{aligned}
$$

The modulus of the latter integral can be estimated by

$$
\|\psi\|_{\infty} \frac{1}{R} \iint_{\operatorname{supp} \psi} \int \sqrt{1+|p|^{2}}\left(f_{n}+f\right) d p d x d t \leq \frac{C}{R}
$$


via the uniform bound on the kinetic energy, and since $R>0$ and the test function $\psi$ are arbitrary the assertion for $j$ follows. That local conservation of charge (2.2) and the second of the Maxwell equations (1.2) hold in the sense of distributions is obvious. As to the first of the Maxwell evolution equations let $\psi$ be as above. Then with the abbreviation $\delta_{n}:=d_{n} * d_{n}$,

$$
\iint \delta_{n} * j_{n} \psi d x d t-\iint j \psi d x d t=\iint\left(\delta_{n} * j_{n}-j_{n}\right) \psi d x d t+\iint\left(j_{n}-j\right) \psi d x d t .
$$

The second term converges to zero, and the first can be estimated by

$$
\left\|j_{n}\right\|_{L^{4 / 3}(\operatorname{supp} \psi)}\left\|\delta_{n} * \psi-\psi\right\|_{4}
$$

which converges to zero as well. Hence $\delta_{n} * j_{n} \rightarrow j$ in the sense of distributions. The computation in Section 2 which showed that the constraints (1.3) propagate can be performed in the weak sense, and the proof is complete.

\section{Compactness via momentum averaging}

In the present section we want to show that the weak limit obtained in the previous one satisfies the Vlasov equation in the sense of distributions. Due to the non-linearity in the latter equation this is the crucial problem in the whole proof. We need to show that for any $\phi \in C_{c}^{\infty}\left(\mathbb{R} \times \mathbb{R}^{6}\right)$,

$$
\iiint\left(E_{n}+\widehat{p} \times B_{n}\right) \cdot \partial_{p} \phi f_{n} d p d x d t \rightarrow \iiint(E+\widehat{p} \times B) \cdot \partial_{p} \phi f d p d x d t
$$

possibly after extracting a further subsequence. By a well known density argument it is sufficient to consider test functions which factorize: $\phi(t, x, p)=\phi_{1}(t, x) \phi_{2}(p)$ with test functions $\phi_{1}, \phi_{2}$. The desired convergence will follow once we can show that for any bounded open interval $I \subset \mathbb{R}$, any $S>0$, and any $\psi \in C_{c}^{\infty}\left(\mathbb{R}^{3}\right)$ up to a subsequence,

$$
\int \psi(p) f_{n}(\cdot, \cdot, p) d p \rightarrow \int \psi(p) f(\cdot, \cdot, p) d p \text { strongly in } L^{2}\left(I \times B_{S}\right),
$$

where $B_{S} \subset \mathbb{R}^{3}$ denotes the ball of radius $S$ about the origin. That this is true is the consequence of the velocity-averaging lemma, a version of which was established in [3], cf. also [14], and which for obvious reasons we prefer to call momentum-averaging lemma. We state it here and give a proof in an appendix:

Lemma 5.1. Let $R>0$ and $\psi \in C_{c}^{\infty}\left(B_{R}\right)$. There exists a constant $C>0$ such that for any functions $h, g_{0}, g_{1} \in L^{2}\left(\mathbb{R} \times \mathbb{R}^{3} \times B_{R}\right)$ which satisfy the inhomogeneous transport equation

$$
\partial_{t} h+\widehat{p} \cdot \partial_{x} h=g_{0}+\operatorname{div}_{p} g_{1}
$$

in the sense of distributions we have

$$
\int \psi(p) h(\cdot, \cdot, p) d p \in H^{1 / 4}\left(\mathbb{R} \times \mathbb{R}^{3}\right)
$$

with

$$
\left\|\int \psi(p) h(\cdot, \cdot, p) d p\right\|_{H^{1 / 4}} \leq C\left(\|h\|_{L^{2}\left(\mathbb{R} \times \mathbb{R}^{3} \times B_{R}\right)}+\left\|g_{0}\right\|_{L^{2}(\cdots)}+\left\|g_{1}\right\|_{L^{2}(\cdots)}\right)
$$


Here $H^{1 / 4}$ denotes the usual fractional order Sobolev space defined in terms of the Fourier transform. In the proof one needs to Fourier transform the transport equation with respect to $t$ and $x$ so that it is essential that the equation holds for all $t \in \mathbb{R}$. Since we apply the lemma to the approximating solutions we avoid considerable technical complications by having the latter defined on the whole real line.

In order to prove (5.1) we take an arbitrary open, bounded interval $I \subset \mathbb{R}, \psi \in$ $C_{c}^{\infty}\left(\mathbb{R}^{3}\right)$, and $R>0$ such that $\operatorname{supp} \psi \subset B_{R}$. Moreover, we choose some test function $\zeta \in C_{c}^{\infty}(\mathbb{R})$ such that $0 \leq \zeta \leq 1$ and $\zeta_{\mid I}=1$. Then for $\tilde{f}_{n}(t, x, p):=\zeta(t) f_{n}(t, x, p)$ the Vlasov equation in divergence form (2.1) implies that

$$
\partial_{t} \tilde{f}_{n}+\widehat{p} \cdot \partial_{x} \tilde{f}_{n}=g_{0}+\operatorname{div}_{p} g_{1}
$$

where $g_{0}:=\zeta^{\prime} f_{n}$ and $g_{1}:=\left(E_{n}+\widehat{p} \times B_{n}\right) \zeta f_{n}$. By Proposition 3 the $L^{2}$-norms of the latter functions over the domain $\mathbb{R} \times \mathbb{R}^{3} \times B_{R}$ are bounded, uniformly in $n$. Hence by Lemma 5.1 we conclude that the momentum averages of the functions $\tilde{f}_{n}$, formed with the test function $\psi$, lie in the Sobolev space $H^{1 / 4}\left(\mathbb{R} \times \mathbb{R}^{3}\right)$, and their corresponding $H^{1 / 4}$-norms are bounded, uniformly in $n$. Over the open, bounded set $I \times B_{S}$ with $S>0$ arbitrary this Sobolev space is compactly embedded in $L^{2}$, by choice of $\zeta$ the functions $\tilde{f}_{n}$ coincide with $f_{n}$ on $I \times B_{S}$, and hence along a suitable subsequence Eqn. (5.1) is established.

We conclude that the weak limit $(f, E, B)$ obtained in the previous section satisfies the complete relativistic Vlasov-Maxwell system on $\mathbb{R} \times \mathbb{R}^{3} \times \mathbb{R}^{3}$ in the sense of distributions.

\section{Continuity properties and conservation laws}

In order to be justified in saying that the weak solution $(f, E, B)$ satisfies the initial conditions we need to establish some minimal continuity in $t$. We consider this problem for $f$, the arguments for $E$ and $B$ being very similar. We express $\partial_{t} f_{n}$ via the Vlasov equation in divergence form, integrate in time, multiply the result by a test function $\phi \in C_{c}^{\infty}\left(\mathbb{R}^{6}\right)$ and integrate in $x$ and $p$ to obtain, after an integration by parts:

$$
\begin{aligned}
\iint f_{n}(t, x, p) \phi(x, p) d p d x & =\iint \stackrel{\circ}{f}_{n}(x, p) \phi(x, p) d p d x \\
& +\int_{0}^{t} \iint\left[\widehat{p} \cdot \partial_{x} \phi+\left(E_{n}+\widehat{p} \times B_{n}\right) \cdot \partial_{p} \phi\right] f_{n}(s, x, p) d p d x d s .
\end{aligned}
$$

For $t \in \mathbb{R}$ we define a distribution $\tilde{f}(t) \in \mathcal{D}^{\prime}\left(\mathbb{R}^{6}\right)$ by

$$
\begin{aligned}
\langle\tilde{f}(t), \phi\rangle:= & \iint \stackrel{\circ}{f}(x, p) \phi(x, p) d p d x \\
& +\int_{0}^{t} \iint\left[\widehat{p} \cdot \partial_{x} \phi+(E+\widehat{p} \times B) \cdot \partial_{p} \phi\right] f(s, x, p) d p d x d s .
\end{aligned}
$$

The mapping $t \mapsto \tilde{f}(t) \in \mathcal{D}^{\prime}\left(\mathbb{R}^{6}\right)$ is continuous in the sense of distributions, $\tilde{f}(0)=\stackrel{\circ}{f}$, and one can show that this is a representative of $f$. By a density argument $t \mapsto f(t)$ is also continuous with respect to the weak topology of $L^{q}\left(\mathbb{R}^{6}\right)$ for any $1<q<\infty$. Since for any bounded measurable set $M \subset \mathbb{R}^{6}$ and $s>0$ the negative order Sobolev space $W^{-s, 2}(M)$ is compactly embedded in $L^{2}(M)$ continuity with respect to the negative order Sobolev norm follows. 
We already noted that the total energy can at least not increase, and that local conservation of charge (2.2) holds in the sense of distributions. It is simple to conclude that for almost all $t, \int \rho(t)$ equals some fixed constant, but we assert that this constant really is the initial charge. Indeed, more is true:

Proposition 5. The weak solution obtained above preserves all $L^{q}$-norms of $f(t)$, more precisely, for every $1 \leq q \leq \infty$,

$$
\|f(t)\|_{q}=\|\stackrel{\circ}{f}\|_{q} \text { for } \text { a. } \text { a. } t \in \mathbb{R} .
$$

Moreover, $t \mapsto f(t)$ is a. e. strongly $L^{q}$ continuous in the following sense: There exists a set of continuity $C \subset \mathbb{R}$ such that the Lebesgue measure $\lambda(\mathbb{R} \backslash C)=0,0 \in C$, and for any $q \in] 1, \infty\left[\right.$ the mapping $C \ni t \mapsto f(t) \in L^{q}\left(\mathbb{R}^{6}\right)$ is strongly continuous.

Proof. Since $\partial_{t} \rho_{n}+\operatorname{div} j_{n}=0$ and $\left|j_{n}\right| \leq \rho_{n}$ we have for every $R>0$ and $t>0$,

$$
\begin{aligned}
\frac{d}{d t} \int_{|x|>R+t} \rho_{n}(t) d x & =-\int_{|x|=R+t} \rho_{n}(t) d S_{x}+\int_{|x|>R+t} \partial_{t} \rho_{n}(t) d x \\
& =-\int_{|x|=R+t} \rho_{n}(t) d S_{x}-\int_{|x|>R+t} \operatorname{div} j_{n}(t) d x \\
& =-\int_{|x|=R+t}\left(\rho_{n}(t)+\nu \cdot j_{n}(t)\right) d S_{x} \leq 0
\end{aligned}
$$

where $\nu$ is the outer unit normal of the domain $\{|x|>R+t\}$. The analogous argument works for $t<0$ and the domain $\{|x|>R-t\}$. Hence

$$
\int_{|x|>R+|t|} \rho_{n}(t) d x \leq \int_{|x|>R} \stackrel{\circ}{\rho}_{n} d x, t \in \mathbb{R}, R>0, n \in \mathbb{N},
$$

where $\stackrel{\circ}{\rho}=\int \stackrel{\circ}{f} d p$. We claim that

$$
\int \rho(t) d x=\int \stackrel{\circ}{\rho} d x \text { for a. a. } t \in \mathbb{R} .
$$

Let $\epsilon>0$ be arbitrary. Since $\stackrel{\circ}{\rho}$ is integrable, we can choose $R>0$ such that

$$
\int_{|x|>R} \stackrel{\circ}{\rho} d x<\epsilon .
$$

By the convergence of the initial data and (6.1) we conclude that

$$
\int_{|x|>R+|t|} \rho_{n} d x \leq \int_{|x|>R} \rho_{n} d x<\epsilon
$$

for all $t \in \mathbb{R}$ and all sufficiently large $n \in \mathbb{N}$. Let $A \subset \mathbb{R}$ be measurable and bounded. Then

$$
\begin{aligned}
\int_{A} \int \rho d x d t & \geq \int_{A} \int_{|x| \leq R+|t|} \rho d x d t=\lim _{n \rightarrow \infty} \int_{A} \int_{|x| \leq R+|t|} \rho_{n} d x d t \\
& =\lim _{n \rightarrow \infty} \int_{A}\left[\int \rho_{n} d x-\int_{|x|>R+|t|} \rho_{n} d x\right] d t>\lambda(A)\left[\int \stackrel{\circ}{\rho} d x-\epsilon\right],
\end{aligned}
$$


and for sufficiently large $S>0$ we have by monotone convergence,

$$
\begin{aligned}
\int_{A} \int \rho d x d t & \leq \int_{A} \int_{|x| \leq S} \rho d x d t+\lambda(A) \epsilon \\
& =\lim _{n \rightarrow \infty} \int_{A} \int_{|x| \leq S} \rho_{n} d x d t+\lambda(A) \epsilon \leq \lambda(A)\left(\int \rho d x+\epsilon\right) .
\end{aligned}
$$

This implies that there exists a set $M_{\epsilon} \subset \mathbb{R}$ of measure zero such that

$$
\int \stackrel{\circ}{\rho} d x-\epsilon \leq \int \rho(t) d x \leq \int \stackrel{\circ}{\rho} d x+\epsilon, t \in \mathbb{R} \backslash M_{\epsilon} .
$$

Hence (6.2) holds on $\mathbb{R} \backslash \cup_{k \in \mathbb{N}} M_{1 / k}$. For general $q \in[1, \infty[$ we define

$$
\rho_{q}(t, x):=\int f^{q}(t, x, p) d p, j_{q}(t, x):=\int \widehat{p} f^{q}(t, x, p) d p,
$$

with analogous definitions for $\rho_{q, n}$ and $j_{q, n}$. Since $f$ and $f_{n}$ are bounded, these new densities converge in the same sense as the ones for $q=1$ and the weak limits are indeed the $q$-densities induced by $f$. Moreover, $\partial_{t} \rho_{q, n}+\operatorname{div} j_{q, n}=0$ classically for every $n$, and $\left|j_{q, n}\right| \leq \rho_{q, n}$. Hence exactly the same argument as in the case $q=1$ shows that

$$
\int \rho_{q}(t) d x=\int \stackrel{\circ}{\rho}_{q} d x
$$

for almost all $t \in \mathbb{R}$. If we pick a sequence $q_{k} \rightarrow \infty$ we can choose the exceptional set of measure zero where (6.3) does not hold uniformly in $k$, and passing to the limit shows that the $L^{\infty}$-norm of $f(t)$ is preserved in the same sense.

Combining this with the weak continuity of the map $t \mapsto f(t)$ or rather a suitable representative, the Radon-Riesz Theorem implies that for every $q \in] 1, \infty[$ one can choose a set of continuity $C_{q}$ with the asserted properties, and via two sequences $q_{k}^{+} \rightarrow \infty$ and $q_{k}^{-} \searrow 1$ we obtain a set of continuity $C$ which works for all $\left.q \in\right] 1, \infty[$ simultaneously.

Note that the relativistic nature of the system was exploited in the proof above. We are not aware of a proof of (6.2) in the non-relativistic case.

\section{The result - statement and comments}

Collecting the results of the previous sections we arrive at the following existence result for global weak solutions to the relativistic Vlasov-Maxwell system:

Theorem. For initial data $\stackrel{\circ}{f} \in L_{\text {kin }}^{1} \cap L^{\infty}\left(\mathbb{R}^{6}\right), \stackrel{\circ}{E}, \stackrel{\circ}{B} \in L^{2}\left(\mathbb{R}^{3}\right)$ which satisfy the constraints (1.3) in the sense of distributions there exists a global weak solution of the relativistic Vlasov-Maxwell system, i.e., there exist functions

$$
f \in L^{\infty}\left(\mathbb{R} ; L_{\text {kin }}^{1} \cap L^{\infty}\left(\mathbb{R}^{6}\right)\right), E, B \in L^{\infty}\left(\mathbb{R} ; L^{2}\left(\mathbb{R}^{3}\right)\right), \rho, j \in L^{\infty}\left(\mathbb{R} ; L^{4 / 3}\left(\mathbb{R}^{3}\right)\right)
$$

such that $(f, E, B)$ satisfy (1.1) - (1.3) in the sense of distributions with $\rho, j$ defined in terms of $f$ by (1.4). The function $f$ is a. e. non-negative.

The mappings $t \mapsto f(t), E(t), B(t)$ are (after choosing suitable representatives) continuous with respect to the following topologies: the standard topology in the space of distributions $\mathcal{D}^{\prime}\left(\mathbb{R}^{6}\right)$ or $\mathcal{D}^{\prime}\left(\mathbb{R}^{3}\right)$ respectively, the weak topology of $L^{2}$, and the strong topology of $W^{-s, 2}(M)$ for any $s>0$ and any bounded measurable subset $M$ of $\mathbb{R}^{6}$ or 
$\mathbb{R}^{3}$ respectively. The mapping $t \mapsto f(t)$ is a. e. strongly continuous into any $L^{q}\left(\mathbb{R}^{6}\right)$, $1<q<\infty$, in the sense of Proposition 5. The initial conditions (1.5) are satisfied.

At a. e. time the total energy

$$
\iint \sqrt{1+|p|^{2}} f(t, x, p) d p d x+\frac{1}{8 \pi} \int\left(|E(t, x)|^{2}+|B(t, x)|^{2}\right) d x
$$

is bounded by its value at $t=0$. The total charge is conserved,

$$
\iint f(t) d p d x=\iint \stackrel{\circ}{f} d p d x \text { for a. a. } t \in \mathbb{R},
$$

and the same is true for any $L^{q}$-norm of $f(t), 1 \leq q \leq \infty$.

The following deficiencies of weak solutions are obvious: firstly, uniqueness is not known. Secondly, it is not clear whether energy is conserved and whether $L^{q}$-norms of $f$ are conserved everywhere. Moreover, their continuity with respect to $t$ holds either everywhere in a rather weak sense or only a. e. in the strong $L^{q}$ sense. A less obvious disadvantage is the following: it is not clear that any weak solution in the sense of the theorem can be obtained via the particular regularization which we employed here, and it is conceivable that results for weak solutions depend on the way in which these are constructed.

Appendix: Momentum averaging. To prove Lemma 5.1 we let $\psi \in C_{c}^{\infty}\left(\mathbb{R}^{3}\right)$ with $\operatorname{supp} \psi \subset B_{R}$ and $R>0$. By the usual definition of the fractional order Sobolev spaces via Fourier transforms,

$$
\left\|\int h(\cdot, \cdot, p) \psi(p) d p\right\|_{H^{1 / 4}}^{2} \leq\|\psi\|_{2}^{2}\|\hat{h}\|_{2}^{2}+\iint|I(\tau, \xi)|^{2}\left(|\tau|^{1 / 2}+|\xi|^{1 / 2}\right) d \xi d \tau
$$

where

$$
I(\tau, \xi):=\int \hat{h}(\tau, \xi, p) \psi(p) d p
$$

and $\hat{h}$ denotes the Fourier transform of $h$ with respect to $(t, x)$. Confusion with the notation $\widehat{p}$ seems unlikely. By assumption, $h$ satisfies the transport equation (5.2) on $\mathbb{R} \times \mathbb{R}^{6}$ in the sense of distributions, and hence

$$
i(\tau+\widehat{p} \cdot \xi) \hat{h}=\hat{g}_{0}+\operatorname{div}_{p} \hat{g}_{1} .
$$

This identity is only useful where the factor on the left hand side is away from zero. Hence we let $\zeta \in C_{c}^{\infty}(\mathbb{R})$ be such that

$$
0 \leq \zeta \leq 1, \operatorname{supp} \zeta \subset[-2,2], \zeta_{\mid[-1,1]}=1
$$

and we split the integral (7.2) into the two parts

$$
\begin{aligned}
& I_{1}(\tau, \xi):=\int \hat{h}(\tau, \xi, p) \psi(p) \zeta\left(\frac{\tau+\hat{p} \cdot \xi}{\kappa}\right) d p, \\
& I_{2}(\tau, \xi):=\int \hat{h}(\tau, \xi, p) \psi(p)\left[1-\zeta\left(\frac{\tau+\hat{p} \cdot \xi}{\kappa}\right)\right] d p,
\end{aligned}
$$

where $\kappa>0$ will be chosen appropriately in dependence of $\xi$. 
For almost all $(\tau, \xi) \in \mathbb{R}^{4}$,

$$
\left|I_{1}(\tau, \xi)\right| \leq C\|\hat{h}(\tau, \xi, \cdot)\|_{2}\left(\frac{\kappa}{|\xi|}\right)^{1 / 2} \mathbf{1}_{|\tau| \leq r|\xi|+2 \kappa}
$$

where $r:=R / \sqrt{1+R^{2}}$ as in the rest of the argument, the $L^{2}$-norm with respect to $p$ refers to the ball $B_{R}$ with $R>0$ fixed, and 1 with some subscript is the "indicator function" of the set or condition in the subscript. To see (7.4) we apply the CauchySchwarz inequality and observe that

$$
\int_{B_{R}} \zeta^{2}\left(\frac{\tau+\widehat{p} \cdot \xi}{\kappa}\right) d p \leq \int_{B_{R}} \mathbf{1}_{[-\tau-2 \kappa,-\tau+2 \kappa]}\left(\widehat{p}_{1}|\xi|\right) d p ;
$$

without loss of generality we may assume that $\xi=(|\xi|, 0,0)$. The integral on the right hand side vanishes if $|\tau|>r|\xi|+2 \kappa$, and via a change of variables it can easily be estimated against $\kappa /|\xi|$ which proves (7.4).

For the estimate of $I_{2}$ the basic idea is to use (7.3) and to integrate by parts in the term containing the divergence of $g_{1}$. With the abbreviation

$$
\chi(\tau, \xi, p):=\psi(p)\left[1-\zeta\left(\frac{\tau+\widehat{p} \cdot \xi}{\kappa}\right)\right] \frac{1}{\tau+\widehat{p} \cdot \xi}
$$

it follows that

$$
I_{2}(\tau, \xi)=-i\left(\int \hat{g}_{0}(\tau, \xi, p) \chi(\tau, \xi, p) d p-\int \hat{g}_{1}(\tau, \xi, p) \cdot \partial_{p} \chi(\tau, \xi, p) d p\right)
$$

where this last identity holds a. e. on $\mathbb{R} \times \mathbb{R}^{3}$ if $\kappa=1$ and a. e. on $\mathbb{R} \times\{|\xi|>1\}$ if $\kappa=|\xi|^{1 / 2}$. The first choice will be used for small $|\xi|$ and the second one for large $|\xi|$. For any multi-index $\alpha \in \mathbb{N}^{3}$ with length $|\alpha| \leq 1$,

$$
\left|D_{p}^{\alpha} \chi(\tau, \xi, p)\right| \leq C \mathbf{1}_{|p| \leq R} \mathbf{1}_{|\tau+\widehat{p} \cdot \xi| \geq \kappa} \frac{1}{|\tau+\widehat{p} \cdot \xi|}\left(1+\frac{|\xi|}{\kappa}\right) .
$$

Hence

$$
\left\|D_{p}^{\alpha} \chi(\tau, \xi, \cdot)\right\|_{2} \leq C\left(1+\frac{|\xi|}{\kappa}\right) \frac{1}{|\xi|^{1 / 2}}\left(\int_{\tau-r|\xi|}^{\tau+r|\xi|} \mathbf{1}_{|w| \geq \kappa} \frac{1}{w^{2}} d w\right)^{1 / 2} .
$$

The remaining integral is straightforwardly estimated by $C / \kappa$. In addition, for $|\tau| \geq$ $r|\xi|+2 \kappa$ the origin does not lie in the domain of integration of this integral, and we can estimate it by integrating $1 / w^{2}$ over the domain. Hence,

$$
\left\|D_{p}^{\alpha} \chi(\tau, \xi, \cdot)\right\|_{2} \leq C\left(1+\frac{|\xi|}{\kappa}\right)\left(\mathbf{1}_{|\tau| \leq r|\xi|+2 \kappa} \frac{1}{\kappa|\xi|}+\mathbf{1}_{|\tau|>r|\xi|+2 \kappa} \frac{1}{\tau^{2}-r^{2}|\xi|^{2}}\right)^{1 / 2}
$$

Let us abbreviate

$$
N(\tau, \xi):=\|\hat{h}(\tau, \xi, \cdot)\|_{2}+\left\|\hat{g}_{0}(\tau, \xi, \cdot)\right\|_{2}+\left\|\hat{g}_{1}(\tau, \xi, \cdot)\right\|_{2} .
$$

Combining (7.4) with (7.5) and the estimate for $D_{p}^{\alpha} \chi$ we finally obtain the following estimate for $I$,

$$
\begin{aligned}
|I(\tau, \xi)| \leq C N(\tau, \xi)\left[\mathbf{1}_{|\tau| \leq r|\xi|+2 \kappa}\left(\frac{\kappa}{|\xi|}+\left(1+\frac{|\xi|}{\kappa}\right)^{2} \frac{1}{\kappa|\xi|}\right)\right. \\
\left.\mathbf{1}_{|\tau| \geq r|\xi|+2 \kappa}\left(1+\frac{|\xi|}{\kappa}\right)^{2} \frac{1}{\tau^{2}-r^{2}|\xi|^{2}}\right]^{1 / 2}
\end{aligned}
$$


which holds a. e. on $\mathbb{R} \times \mathbb{R}^{3}$ if $\kappa=1$ and a. e. on $\mathbb{R} \times\{|\xi|>1\}$ if $\kappa=|\xi|^{1 / 2}$. Hence for $|\xi| \leq 1$ we take $\kappa=1$ to obtain

$$
|I(\tau, \xi)| \leq C N(\tau, \xi)\left(\mathbf{1}_{|\tau| \leq r|\xi|+2} \frac{1}{|\xi|}+\mathbf{1}_{|\tau| \geq r|\xi|+2} \frac{1}{\tau^{2}-r^{2}|\xi|^{2}}\right)^{1 / 2}
$$

and for $|\xi|>1$ we take $\kappa=|\xi|^{1 / 2}$ to obtain

$$
|I(\tau, \xi)| \leq C N(\tau, \xi)\left(\mathbf{1}_{|\tau| \leq r|\xi|+2|\xi|^{1 / 2}} \frac{1}{|\xi|^{1 / 2}}+\mathbf{1}_{|\tau| \geq r|\xi|+2|\xi|^{1 / 2}} \frac{|\xi|}{\tau^{2}-r^{2}|\xi|^{2}}\right)^{1 / 2} .
$$

Now we split the right hand side of (7.1) as follows:

$$
\begin{aligned}
& \iint|I(\tau, \xi)|^{2}\left(|\tau|^{1 / 2}+|\xi|^{1 / 2}\right) d \tau d \xi=\iint|I|^{2}|\tau|^{1 / 2} \mathbf{1}_{|\xi|>1} d \tau d \xi \\
& \quad+\iint|I|^{2}|\tau|^{1 / 2} \mathbf{1}_{|\xi| \leq 1} \mathbf{1}_{|\tau|>r+2} d \tau d \xi+\iint|I|^{2}|\tau|^{1 / 2} \mathbf{1}_{|\xi| \leq 1} \mathbf{1}_{|\tau| \leq r+2} d \tau d \xi \\
& \quad+\iint|I|^{2}|\xi|^{1 / 2} \mathbf{1}_{|\xi| \leq 1} d \tau d \xi+\iint|I|^{2}|\xi|^{1 / 2} \mathbf{1}_{|\xi|>1} d \tau d \xi \\
& =: A_{1}+A_{2}+A_{3}+A_{4}+A_{5} .
\end{aligned}
$$

Using the appropriate part of the estimate for $I$ we find that

$$
A_{1}+A_{2}+A_{5} \leq C \iint N^{2} d \tau d \xi=C\left(\|\hat{h}\|_{2}^{2}+\left\|\hat{g}_{0}\right\|_{2}^{2}+\left\|\hat{g}_{1}\right\|_{2}^{2}\right)
$$

as desired, while the terms $A_{3}$ and $A_{4}$ can be estimated directly by $\|\hat{h}(\tau, \xi, \cdot)\|_{2}^{2}$ via the Cauchy-Schwarz inequality, and the proof of Lemma 5.1 is complete.

Acknowledgment. The present notes originate in a seminar on kinetic theory at the University of Vienna. I would like to thank the participants of that seminar as well as Prof. R. T. Glassey for their interest in the simplifications I report here.

\section{REFERENCES}

[1] F. Bouchut, F. Golse, and C. Pallard, On classical solutions to the 3d relativistic Vlasov-Maxwell system: Glassey-Strauss' theorem revisited, Preprint, 2003, arXiv:math.AP/0301175v1.

[2] S. Calogero and G. Rein, Global weak solutions to the Nordström-Vlasov system, J. Differential Eqns., to appear.

[3] R.J. DiPerna and P.L. Lions, Global weak solutions of Vlasov-Maxwell systems, Commun. Pure Appl. Math., 42(6):729-757, 1989.

[4] R.J. DiPerna and P.L. Lions, On the Cauchy problem for Boltzmann equations; global existence and weak stability, Ann. Math., 130:321-366, 1989.

[5] R.T. Glassey, The Cauchy Problem in Kinetic Theory, SIAM, Philadelphia, 1996.

[6] R.T. Glassey and J. Schaeffer, On symmetric solutions of the relativistic Vlasov-Poisson system, Commun. Math. Phys., 101:459-473, 1985.

[7] R.T. Glassey and J. Schaeffer, Global existence for the relativistic Vlasov-Maxwell system with nearly neutral data, Commun. Math. Phys., 119:353-384, 1988.

[8] R.T. Glassey and J. Schaeffer, Control of velocities generated in a two-dimensional collisionless plasma with symmetry, Transp. Theory and Stat. Mech., 17:467-560, 1988.

[9] R.T. Glassey and J. Schaeffer, On the "one and one-half dimensional" relativistic VlasovMaxwell system, Math. Meth. Appl. Sci., 13:169-179, 1990.

[10] R.T. Glassey and J. Schaeffer, The "two and one-half dimensional" relativistic Vlasov-Maxwell system, Commun. Math. Phys., 185:257-284, 1997. 
[11] R.T. Glassey and J. Schaeffer, The relativistic Vlasov-Maxwell system in two space dimensions: Parts I $\& 3$ II, Arch. Rational Mech. Anal., 141:331-354 \& 355-374, 1998.

[12] R.T. Glassey and W. Strauss, Singularity formation in a collisionless plasma could occur only at high velocities, Arch. Rat. Mech. Anal., 92:59-90, 1986.

[13] R.T. Glassey and W. Strauss, Absence of shocks in an initially dilute collisionless plasma, Commun. Math. Phys., 113:191-208, 1987.

[14] F. Golse, P.L. Lions, B. Perthame, and R. Sentis, Regularity of the moments of the solution of a transport equation, J. Funct. Anal., 76(1):110-125, 1988.

[15] E. Horst, Global solutions of the relativistic Vlasov-Maxwell system of plasma physics, Dissertationes Mathematicae, CCXCII:1-63, 1990.

[16] S. Klainerman and G. Staffilani, A new approach to study the Vlasov-Maxwell system, Commun. Pure Appl. Anal., 1:103-125, 2002.

[17] K. Kruse, Ein neuer Zugang zur globalen Existenz von Distributionenlösungen des VlasovMaxwell-Systems partieller Differentialgleichungen, Diploma thesis, University of Munich, 1991.

[18] K. Kruse and G. Rein, A stability result for the relativistic Vlasov-Maxwell system, Arch. Rational Mech. Anal., 121(2):187-203, 1992.

[19] M. Kunzinger, G. Rein, R. Steinbauer, and G. Teschl, Global weak solutions of the relativistic Vlasov-Klein-Gordon system, Commun. Math. Phys., 238(1-2):367-378, 2003.

[20] P.L. Lions and B. Perthame, Propagation of moments and regularity for the 3-dimensional Vlasov-Poisson system, Invent. Math., 105:415-430, 1991.

[21] K. Pfaffelmoser, Global classical solutions of the Vlasov-Poisson system in three dimensions for general initial data, J. Diff. Eqns., 95:281-303, 1992.

[22] G. Rein, Generic global solutions of the relativistic Vlasov-Maxwell system of plasma physics, Commun. Math. Phys., 135:41-78, 1990.

[23] J. Schaeffer, Global existence of smooth solutions to the Vlasov-Poisson system in three dimensions, Commun. Part. Diff. Eqns., 16:1313-1335, 1991. 